\title{
Helical liquid of snake states
}

\author{
Kh. Shakouri, S. M. Badalyan, ${ }^{*}$ and F. M. Peeters \\ Department of Physics, University of Antwerp, Groenenborgerlaan 171, B-2020 Antwerpen, Belgium \\ (Received 31 May 2013; revised manuscript received 12 October 2013; published 7 November 2013)
}

\begin{abstract}
We derive an exact solution to the problem of spin snake states induced in a nonhomogeneous magnetic field by a combined action of the Rashba spin-orbit and Zeeman fields. The electron spin behavior as a function of the cyclotron orbit center position and an external homogeneous magnetic field was obtained. It is shown that in an antisymmetric magnetic field the electron spin in the snake states has only an in-plane projection, perpendicular to the magnetic interface, which vanishes at large positive momenta. Applying an external homogeneous magnetic field adds a finite out-of-plane spin component and simultaneously gaps out the spectral branches, which results in regular beating patterns of the spin current components.
\end{abstract}

DOI: 10.1103/PhysRevB.88.195404

PACS number(s): 73.43.-f, 71.70.Ej, 85.75.-d

\section{INTRODUCTION}

In a spatially nonhomogeneous magnetic field, which changes its sign across a magnetic interface, the Lorenz force produces opposite cyclotron rotations of electrons on both sides of the interface, resulting in a drift of carriers in snakelike classical trajectories. Gapless excitations of snake states are formed, leading to conduction along the magnetic interface. The snake states have been a subject of active theoretical ${ }^{1-4}$ and experimental ${ }^{5-7}$ investigations in two-dimensional electron systems (2DES) formed in semiconductor heterojunctions as well as in graphene. ${ }^{8-10}$ In a homogeneous magnetic field the snake states are formed also along the lines separating electrons and holes and play a key role in transport near the charge neutrality point of topological insulators ${ }^{11,12}$ and graphene. ${ }^{13-15}$

Spin-orbit interaction (SOI) provides an effective tool for electrical control of the spin behavior. ${ }^{16}$ It enables physical separation of the spin of charge carriers, ${ }^{17-20}$ permitting spin transport properties in a non-Ohmic regime. Helical one-dimensional states with opposite spins moving in opposite directions can be effectively generated by a combined action of SOI and a homogeneous magnetic field in quantum wires ${ }^{21-23}$ and at edges of 2DES. ${ }^{24-26}$ Suppression of backscattering processes and large interedge states equilibration length ${ }^{27,28}$ make such spin edge states suitable for spintronics applications, ${ }^{29,30}$ particularly for quantum information processing purposes. ${ }^{31,32}$ As a distinction from spin edge states, the helical system of snake states exhibits unidirectional motion, which emerges due to the spatially nonhomogeneous magnetic field, and which in combination with SOI can produce additional spin transport channels with even richer physics.

Here we present an exact solution of the problem of spin snake states that emerge due to the combined action of the Rashba spin-orbit and Zeeman fields in the presence of a magnetic interface, making use of the method developed in Ref. 19. Our theory is equally applicable to the spin snakes formed along $\mathrm{p}-\mathrm{n}$ junctions in a homogeneous magnetic field. We find that the energy dispersions are described by the main Landau number, the spin-parity and the spin-chirality quantum numbers. In an antisymmetric magnetic field the spin-split quasi-bulk Landau levels are degenerate with respect to the spin parity. Close to the magnetic interface this degeneracy is lifted except the point where the even and odd spin- parity terms cross. We demonstrate that for such a magnetic field profile the average electron spin has only a projection, perpendicular to the magnetic interface. Moreover, this spin component vanishes for states with large positive momenta, which implies that electrons in such spin snake states execute semiclassical cyclotron orbits with opposite rotations and opposite spins on both sides of the magnetic interface. When an additional homogeneous magnetic field is applied, moving the total averaged magnetic field slightly away from zero, the spin-parity symmetry is broken and we find that the average electron spin exhibits a jump, acquiring a finite out-of-plane component. Towards the magnetic interface the in-plane $x$ component (perpendicular to the interface) of the electron spin becomes finite while the out-of-plane $z$ component decreases. Both spin projections show an oscillating behavior before the $z$ component will tend to zero and the $x$ component to a finite value. This is consistent with changes of the Rashba field in parallel to the transformation of the electron circular motion into an unidirectional one along the magnetic interface. Furthermore, at the energies corresponding to the crossings in the spectrum of the spin snake states, we find strong peaks in the spin current. Applying an external uniform magnetic field gaps out the electronic spectral branches and produces a helical liquid of spin snake states resulting in regular beating patterns in the spin current components. These predictions of the properties of spin snake states can be used in an experimental probe of this special class of helical liquid and be of practical relevance for spintronic applications.

\section{THEORETICAL CONCEPT}

Consider a 2DES formed in a quantum well parallel to the (001) plane of a zinc-blende semiconductor heterostructure. A nonhomogeneous antisymmetric magnetic field, $\operatorname{sign}(x) B_{0}$, together with an external uniform field, $B_{u}$, is applied perpendicular to the 2DES. In the presence of the Rashba spin-orbit and Zeeman fields, the effective Hamiltonian of the system can be written as

$$
H=\frac{\vec{\pi}^{2}}{2 m^{*}} \mathbb{1}+\alpha\left[\pi_{x} \hat{\sigma}_{y}-\pi_{y} \hat{\sigma}_{x}\right]-\frac{1}{2} g \mu_{B} B(x) \hat{\sigma}_{z} .
$$

Here $m^{*}$ is the electron effective mass, $\vec{\pi}$ the kinetic momentum, $\mathbb{1}$ and $\hat{\sigma}_{x, y, z}$ the unit and Pauli matrices in spin space, $\mu_{B}$ the Bohr magneton, $\alpha$ and $g$ the strength of Rashba SOI and the 
Landè factor, respectively. In the Landau gauge with the vector potential $\vec{A}=[0, x B(x)]$ where $B(x)=B_{u}+\operatorname{sign}(x) B_{0}$ is the total magnetic field, the Hamiltonian (1) is invariant under translations along the $y$ direction and we can reduce the two-dimensional Schrödinger equation into an effective one-dimensional problem making use of the ansatz $\Psi(x, y)=$ $e^{i k_{y} y} \chi_{k_{y}}(x)$. Here $k_{y}$ is the electron wave vector and $\chi_{k_{y}}(x)=$ $\left|\chi_{1 k_{y}}(x), \chi_{2 k_{y}}(x)\right|^{T}$ the spinor wave function. We introduce dimensionless quantities for the $x$ coordinate, $\sqrt{2} x / l_{B}$, and for the center of cyclotron orbit, $X\left(k_{y}\right)=\sqrt{2} k_{y} l_{B}$, in units of the magnetic length, $l_{B}=\sqrt{\hbar / e B}$, and for the electron energy, $\varepsilon / \hbar \omega_{B}=v+1 / 2$, in units of the cyclotron frequency, $\omega_{B}=e B / m^{*}$. Here $B=B_{u}+B_{0}$ and it is assumed that $B_{u}, B_{0}>0$. Then we can cast the Schrödinger equation for $\chi_{k_{y}}(x)$ in the following compact matrix form

$$
\left(\begin{array}{ll}
h_{\nu \pm \lambda} & \pm h_{ \pm} \\
\pm h_{\mp} & h_{\nu \mp \lambda}
\end{array}\right)\left(\begin{array}{c}
\chi_{1 k_{y}}(\xi) \\
\chi_{2 k_{y}}(\xi)
\end{array}\right)=0
$$

where $\xi=x \sqrt{\theta(x)} \mp X\left(k_{y}\right) / \sqrt{\theta(x)}$ with the upper (lower) sign corresponding to $x>0(x<0)$. The step function $\theta(x)=$ 1 for $x>0$ and $\theta(x)=\left|B_{u}-B_{0}\right| /\left(B_{u}+B_{0}\right)$ for $x<0$. The operators in Eq. (2) are defined as

$$
\begin{aligned}
h_{\nu \pm \lambda} & =\frac{d^{2}}{d \xi^{2}}+\frac{1}{\theta(x)}\left(v+\frac{1}{2}\right) \pm \lambda-\frac{\xi^{2}}{4}, \\
h_{ \pm} & =-\frac{\gamma}{\sqrt{\theta(x)}}\left(\frac{\xi}{2} \mp \frac{d}{d \xi}\right),
\end{aligned}
$$

where the dimensionless parameters $\gamma=2 \alpha / v_{B} \quad\left(v_{B}=\right.$ $\sqrt{2} l_{B} \omega_{B}$ is the cyclotron velocity) and $\lambda=g \mu_{B} m^{*} / 2 e \hbar$ describe the spin-orbit and Zeeman fields, respectively, and $\gamma$ depends on the magnetic field strength.
In the absence of SOI, $h_{ \pm}=0$, the solution of Eq. (2) is given in terms of the scalar parabolic cylindrical functions, $D_{v}(x){ }^{4}$ For $v \geqslant-1 / 2$ and $v \neq 0,1,2, \ldots$ there exist two independent solutions, $D_{v}(x)$ and $D_{v}(-x)$ and $D_{v}(x) \rightarrow 0$ only for $x \rightarrow+\infty$. Making use of this behavior, we construct the solution of the matrix equation (2) satisfying the boundary conditions at infinity in terms of the following spinors

$$
\chi_{ \pm}^{L}(\xi)=\left(\begin{array}{c}
c_{ \pm}^{L} D_{\mu_{ \pm}^{L}-1}\left(-\xi^{L}\right) \\
D_{\mu_{ \pm}^{L}}\left(-\xi^{L}\right)
\end{array}\right), \quad \chi_{ \pm}^{R}(\xi)=\left(\begin{array}{c}
D_{\mu_{ \pm}^{R}}\left(\xi^{R}\right) \\
c_{ \pm}^{R} D_{\mu_{ \pm}^{R}-1}\left(\xi^{R}\right)
\end{array}\right)
$$

which represent the independent bulk solutions of Eq. (2), exhibiting single particle peaks on the left and right hand sides of the magnetic interface for both \pm signs, when

$$
\begin{aligned}
\mu_{ \pm}^{L, R} & =\frac{1}{\theta(x)}\left(v+\frac{1}{2}+\frac{\gamma^{2}}{2} \pm \sqrt{\mathcal{D}}\right), \\
c_{ \pm}^{L, R} & =-\frac{\sqrt{\theta(x)}}{\gamma}\left[\frac{1}{2}-\lambda+\frac{1}{\theta(x)}\left(\frac{\gamma^{2}}{2} \pm \sqrt{\mathcal{D}}\right)\right] .
\end{aligned}
$$

Here $\mathcal{D}=\gamma^{2}\left(v+1 / 2+\gamma^{2} / 4\right)+\theta^{2}(x)(1 / 2-\lambda)^{2}$ and the index $L(R)$ refers to the $x<0(x>0)$ values of respective quantities. To satisfy the boundary conditions at the magnetic interface and to obtain the spectrum of spin snake states, we form a linear combination of the independent bulk solutions (4) as

$$
\psi(x)=a_{L} \chi_{+}^{L}(\xi)+b_{L} \chi_{-}^{L}(\xi)+a_{R} \chi_{+}^{R}(\xi)+b_{R} \chi_{-}^{R}(\xi)
$$

and obtain the coefficients $a_{L, R}$ and $b_{L, R}$ from the continuity of the new spinor wave function $\psi(x)$ and its derivative $\psi^{\prime}(x)$ at $x=0$. This $4 \times 4$ eigenvalue problem has a nontrivial solution if the respective determinant vanishes, from which we obtain the exact dispersion equation for the spin snake states

$$
\operatorname{det}\left|\begin{array}{cccc}
D_{\mu_{+}^{R}}\left(\xi^{R}\right) & D_{\mu_{-}^{R}}\left(\xi^{R}\right) & -c_{+}^{L} D_{\mu_{+}^{L}-1}\left(-\xi^{L}\right) & -c_{-}^{L} D_{\mu_{-}^{L}-1}\left(-\xi^{L}\right) \\
c_{+}^{R} D_{\mu_{+}^{R}-1}\left(\xi^{R}\right) & c_{-}^{R} D_{\mu_{-}^{R}-1}\left(\xi^{R}\right) & -D_{\mu_{+}^{L}}\left(-\xi^{L}\right) & -D_{\mu_{-}^{L}}\left(-\xi^{L}\right) \\
D_{\mu_{+}^{R}}^{\prime}\left(\xi^{R}\right) & D_{\mu_{-}^{R}}^{\prime}\left(\xi^{R}\right) & -c_{+}^{L} D_{\mu_{+}^{L}-1}^{\prime}\left(-\xi^{L}\right) & -c_{-}^{L} D_{\mu_{-}^{L}-1}^{\prime}\left(-\xi^{L}\right) \\
c_{+}^{R} D_{\mu_{+}^{R}-1}^{\prime}\left(\xi^{R}\right) & c_{-}^{R} D_{\mu_{-}^{R}-1}^{\prime}\left(\xi^{R}\right) & -D_{\mu_{+}^{L}}^{\prime}\left(-\xi^{L}\right) & -D_{\mu_{-}^{L}}^{\prime}\left(-\xi^{L}\right)
\end{array}\right|_{x=0}=0 .
$$

The energy dispersions $\varepsilon_{l p q}$ are described by the main Landau quantum number $l=0,1,2, \ldots$ and the spin-parity, $p=$ \pm 1 , and spin-chirality, $q= \pm 1$, quantum numbers. In an antisymmetric magnetic field $\left(B_{u}=0\right)$ the electron system coupled with SOI, possesses a spin-parity symmetry and the eigenvalue problem reduces to two $2 \times 2$ matrix equations.

\section{RESULTS AND DISCUSSIONS}

\section{A. Energy spectrum of spin snake states}

Using Eq. (7) we carry out numerical calculations of the spectrum of spin snake states in 2DES residing in InAs quantum wells for realistic values of the parameters $\hbar \alpha=$ $112.49 \mathrm{meV} \AA, g=-14.8$, and $m^{*}=0.026 m_{e}$ where $m_{e}$ is the free electron mass. Notice that both the Rashba and Zeeman fields can be significant in these structures, however, in relatively weak magnetic fields the SOI dominates, i.e., $\gamma \gg \lambda$.

In a real experiment an abrupt variation of the magnetic field was realized by depositing a ferromagnetic stripe above an electron gas that by the application of a magnetic field parallel to the 2DES and perpendicular to the stripe produces a perpendicular component of the magnetization underneath the stripe (see, e.g., Ref. 7). The obtained exact profile of the magnetic field at low temperatures can be adequately approximated by a magnetic step with two levels of positive and negative magnetic fields $B \lesssim 0.5 \mathrm{~T}^{7}$

Figure 1 shows the energy spectrum of snake states, $\varepsilon_{l p q}\left(k_{y}\right)$, as a function of the cyclotron orbit center position $X\left(k_{y}\right)$. Introducing the spin-orbit and the Zeeman fields in the presence of a magnetic interface removes the degeneracy of the usual Landau structure in a specific way. Groups of 

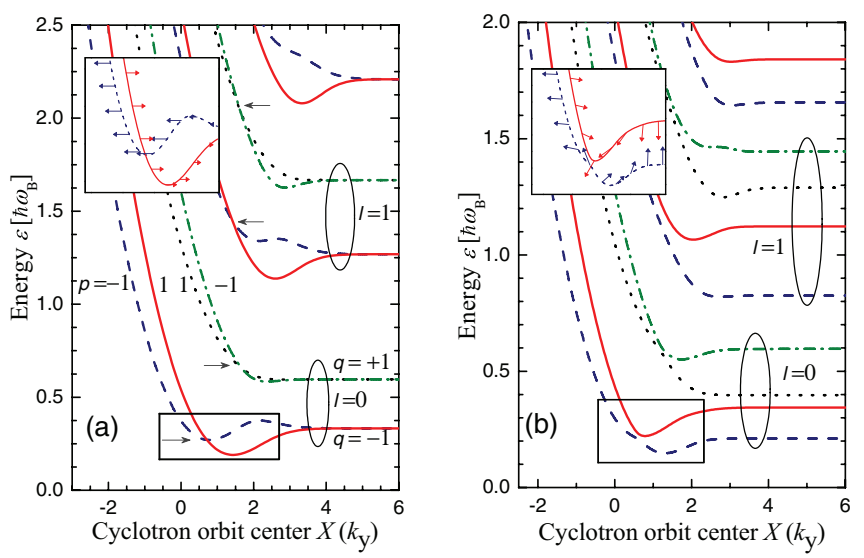

FIG. 1. (Color online) Energy dispersions of spin snake states as a function of the position of the cyclotron orbit center for: (a) $B_{u}=0$ and (b) $B_{u}=0.2 B_{0}$. For given Landau number $l=0,1,2 \ldots$ the four energy branches are described by the spin-parity, $p= \pm 1$, and the spin-chirality, $q= \pm 1$, quantum numbers and are depicted, respectively, by solid, dashed, dotted, and dash-dotted curves. Insets are zooms of the open rectangles. In the main figure the arrows indicate the crossing points of the even and odd spin-parity energy terms while in the insets the arrows depict the electron average spin. The spin-orbit and Zeeman field parameters are $\gamma \approx 0.3$ (for $B_{0} \approx 0.2 \mathrm{~T}$ and $\left.B_{u}=0\right)$ and $\lambda \approx 0.1$.

four different energy levels are formed for given main Landau number $l$. In an antisymmetric magnetic field [cf. Fig. 1(a)] the energy branches far from the magnetic interface represent the spin-split quasibulk Landau levels, which are described by the $q= \pm 1$ spin chirality for given $l$. The splitting energy is determined by the strength of the spin-orbit and Zeeman fields. Each quasibulk Landau level remains partially degenerate due to the spin-parity symmetry. Usually, a magnetic field breaks the spin parity, ${ }^{22}$ here, however, the combined action of the magnetic interface and the SOI preserves the spin parity in the bulk. Close to the magnetic interface, snake orbits with even $(p=1)$ and odd $(p=-1)$ spin parities start to hybridize, which removes the degeneracy of the spin snake states except in one singular point where the even and odd terms cross. The application of an external uniform magnetic field [cf. Fig. 1(b)] brakes the spin-parity symmetry - the singular crossing points are converted into anticrossings and the branches of the spectrum become fully spin-resolved. In this case, the energy of the quasibulk Landau levels is determined by the two different cyclotron frequencies, ${ }^{33,34}$ and this is an extra source of asymmetry in the spectrum.

\section{B. Average spin of snake states}

The combined action of the magnetic interface and the spin interactions has critical consequences for the behavior of the electron average spin projections, $S_{x, z}\left(k_{y}\right)=$ $\int d x \psi^{+}(x) \hat{\sigma}_{x, z} \psi(x)$, which are calculated using the energy dispersions $\varepsilon_{l p q}\left(k_{y}\right)$. We observe in Figs. 2(a) and 2(c) that in an antisymmetric magnetic field $\left(B_{u}=0\right)$ the average spin vanishes in the bulk at large positive values of the momentum for any values of the spin-parity and the spin-chirality quantum numbers. The $S_{x}$ projection of the spin vanishes in the limit of $X\left(k_{y}\right) \rightarrow+\infty$ because of the electron circular motion.
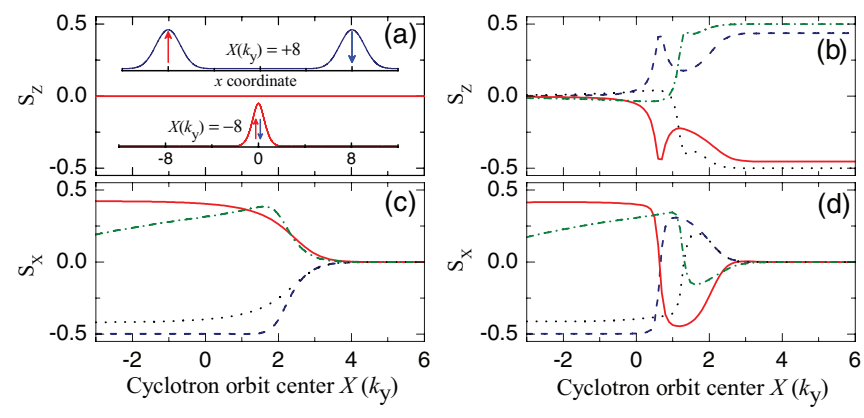

FIG. 2. (Color online) The $S_{z}$ and $S_{x}$ spin projections of the snake states for the lowest $l=0$ Landau band as a function of the position of the cyclotron orbit center. (a) and (c) are for $B_{u}=0$, and (b) and (d) are for $B_{u}=0.2 B_{0}$. In (a) the upper (lower) inset shows the charge density profile of the snake state versus the $x$ coordinate in units of $l_{B} / \sqrt{2}$ for the cyclotron center position at $X\left(k_{y}\right)=+8\left(X\left(k_{y}\right)=-8\right)$. Arrows show the direction of the spin $z$ component in the respective charge density profiles. The other parameters and the type of curves corresponding to the $p= \pm 1$ and $q= \pm 1$ states are the same as in Fig. 1 .

The snake state exhibits a spatially separated double peak (a single peak) structure in its charge density profile across the magnetic interface for positive (negative) values of $k[c f$. the upper (lower) inset in Fig. 2(a)]. We find that the real spins of the pure states (4) with the isospin indices $L$ and $R$, corresponding to the magnetic field sign on the lhs and rhs of the magnetic interface, are linked together in the formation of the spin snake state in such a way that it has a vanishing $S_{z}$ component of the spin [cf. Fig. 2(a)]. This correlation is due to the combined effect of the magnetic interface and SOI and occurs even for arbitrary large positive values of $k$ when correlations between the charge density peaks disappear. Thus, the presented exact solution in an antisymmetric magnetic field describes the electron dynamics in spin snake states with opposite cyclotron rotations and anticorrelated spins on both sides of the magnetic interface.

The application of an external homogeneous magnetic field destroys this spin-parity symmetry, and the $S_{z}$ projection of the spin jumps from zero to a finite value. This jump is strongly pronounced at the crossing points, $X_{c}$, of the energy branches with the even and odd spin parity $[c f$. the peaked behavior of $S_{z}$ at $X_{c} \approx 0.71$ for the solid and dashed curves in Fig. 2(b)]. In the opposite limit of $X\left(k_{y}\right) \rightarrow-\infty$ the snake states exhibit a unilateral motion along the magnetic interface and $S_{z}$ vanishes. As seen in Fig. 2(c), being zero in the bulk the $S_{x}$ spin projection becomes finite when the electron moves towards the interface. This behavior is modified in a finite external homogeneous field [ $c f$. Fig. 2(d)]. It is seen that close to the interface $S_{x}$ starts to oscillate before reaching the finite value. Notice also that the orientation of $S_{x}$ is independent of the direction of the total averaged magnetic field, which determines, however, the orientation of the $S_{z}$ projection.

\section{Spin current carried by snake states}

An external homogeneous magnetic field brakes the spinparity symmetry, removing not only the degeneracy of the quasibulk $p= \pm 1$ Landau levels with a vanishing velocity 


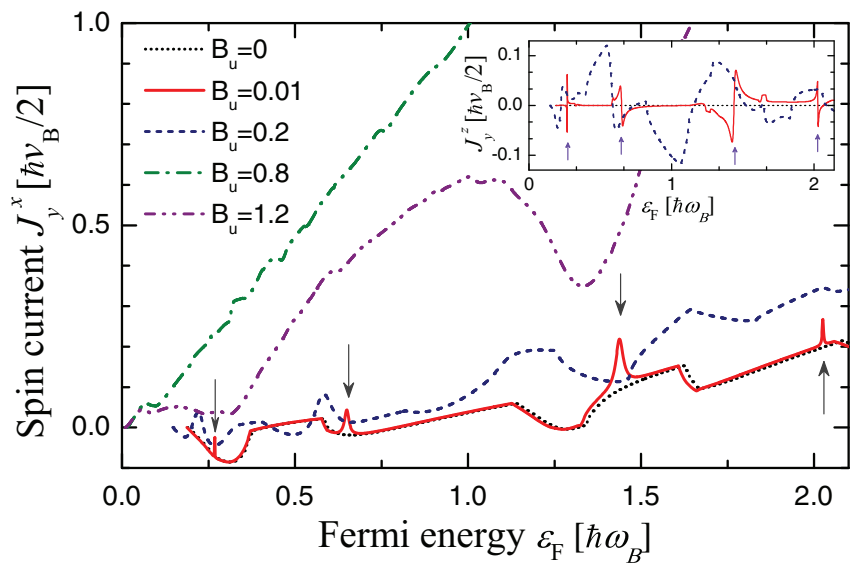

FIG. 3. (Color online) The $J_{y}^{x}\left(J_{y}^{z}\right)$ current of the $x(z)$ spin projection along the magnetic interface shown as a function of the Fermi energy in the main figure (in the inset). The dotted, solid, dashed, dot-dashed, and dot-dot-dashed curves are calculated, respectively, for $B_{u}=0,0.01,0.2,0.8$, and $1.2 B_{0}$. The other parameters are the same as in Fig. 1. The arrows show the positions of the local beatings and peaks, corresponding to the crossings in the spectrum of spin snake states in Fig. 1(a).

but, what is more important for transport, gapping out spectral branches of the extended snake states at the crossing points $X_{c}$ close to the magnetic interface ( $c f$. the insets of Fig. 1). Thus, the combined effect of the magnetic interface and the spin interactions induces a helical liquid of spin snake orbits with additional channels for carrying an uncompensated spin current. The overall spin current is calculated from $J_{y}^{x, z}=\left.\sum S_{x, z}\left(k_{y}\right) v_{y}\left(k_{y}\right)\right|_{\varepsilon_{l p q}\left(k_{y}\right)=\varepsilon_{F}}$ where the sum is over the quantum numbers of current carrying spin snake channels at the Fermi energy $\varepsilon_{F}$ and the electron group velocity along the magnetic interface is $v_{y}\left(k_{y}\right)=\partial \varepsilon_{l p q}\left(k_{y}\right) / \partial k_{y}$. We use this definition of spin current, which is intuitive from a physical point of view and is widely accepted. ${ }^{35,36}$ We calculate the spin current at $T=0$. At finite but low temperatures the predicted structures in the spin current will be broader and smoother but still pronounced. In Fig. 3 we observe that the application of an external uniform magnetic field induces local peaks and beatings, respectively, in the current of the $S_{x}$ and $S_{z}$ spin projections versus the Fermi energy (compare the curves corresponding to $B_{u}=0$ and $B_{u}=0.01 B_{0}$ in the main figure for $J_{y}^{x}$ and in the inset for $J_{y}^{z}$ ), which appear precisely at the crossing points of the energy levels with even and odd spin parity. Note that the charge current does not exhibit such beatings or peaks. When increasing the applied uniform field, the shape of the energy dispersion changes and the peaks are broadened. As seen in Fig. 3, for $0<B_{u}<B_{0}$ the overall magnitude of $J_{y}^{x}$ increases monotonically with $B_{u}$. For $B_{u}=0.8 B_{0}$ it shows almost a linear dependence on the Fermi energy because the velocity of extended snake orbits is linear in $\varepsilon_{F}$ while the $x$ component of the average spin $S_{x}$ is approximately directed along the $x$ axis. However, this monotonic tendency in the dependence of $J_{y}^{x}$ versus $B_{u}$ ceases to exist at $B_{u}=B_{0}$. For $B_{u}>B_{0}$ the magnetic field does not change its sign at the magnetic interface, the extended snake orbits are transformed into the spiral orbit states with a smaller velocity and this results in a suppression of the spin current, which is seen in Fig. 3 for $B_{u}=1.2 B_{0}$.

\section{CONCLUSIONS}

In conclusion, we demonstrated that in the presence of Rashba SOI the spin snake states in an antisymmetric magnetic field have zero out-of-plane spin projection, independently of the cyclotron orbit center position. Applying an external uniform magnetic field destroys the spin-parity symmetry, gapping out the spectral branches of the spin snake states with finite velocities. It leads to new structures in the behavior of the spin current components. These predictions are of experimental relevance in spin transport phenomena and have a potential for spintronic applications.

\section{ACKNOWLEDGMENTS}

This work was supported by the Methusalem program of the Flemish government and the Flemish Science Foundation (FWO-Vl). *samvel.badalyan@ua.ac.be

${ }^{1}$ J. E. Müller, Phys. Rev. Lett. 68, 385 (1992).

${ }^{2}$ F. M. Peeters and A. Matulis, Phys. Rev. B 48, 15166 (1993).

${ }^{3}$ F. M. Peeters, J. Reijniers, S. M. Badalian, and P. Vasilopoulos, Microelectron. Eng. 47, 405 (1999).

${ }^{4}$ S. M. Badalyan and F. M. Peeters, Phys. Rev. B 64, 155303 (2001).

${ }^{5}$ H. A. Carmona, A. K. Geim, A. Nogaret, P. C. Main, T. J. Foster, M. Henini, S. P. Beaumont, and M. G. Blamire, Phys. Rev. Lett. 74, 3009 (1995).

${ }^{6}$ P. D. Ye, D. Weiss, R. R. Gerhardts, M. Seeger, K. von Klitzing,

K. Eberl, and H. Nickel, Phys. Rev. Lett. 74, 3013 (1995).

${ }^{7}$ A. Nogaret, S. J. Bending, and M. Henini, Phys. Rev. Lett. 84, 2231 (2000).

${ }^{8}$ L. Oroszlány, P. Rakyta, A. Kormányos, C. J. Lambert, and J. Cserti, Phys. Rev. B 77, 081403(R) (2008).

${ }^{9}$ T. K. Ghosh, A. De Martino, W. Häusler, L. Dell'Anna, and R. Egger, Phys. Rev. B 77, 081404(R) (2008).
${ }^{10}$ A. De Martino, A. Hytten, and R. Egger, Phys. Rev. B 84, 155420 (2011).

${ }^{11}$ G. M. Gusev, E. B. Olshanetsky, Z. D. Kvon, N. N. Mikhailov, S. A. Dvoretsky, and J. C. Portal, Phys. Rev. Lett. 104, 166401 (2010).

${ }^{12}$ G. M. Gusev, E. B. Olshanetsky, Z. D. Kvon, A. D. Levin, N. N. Mikhailov, and S. A. Dvoretsky, Phys. Rev. Lett. 108, 226804 (2012).

${ }^{13}$ J. R. Williams and C. M. Marcus, Phys. Rev. Lett. 107, 046602 (2011).

${ }^{14}$ J.-C. Chen, X. C. Xie, and Q.-F. Sun, Phys. Rev. B 86, 035429 (2012).

${ }^{15}$ M. Barbier, G. Papp, and F. M. Peeters, Appl. Phys. Lett. 100, 163121 (2012)

${ }^{16}$ J. Fabian, A. Matos-Abiague, C. Ertler, P. Stano, and I. Žutić, Acta Phys. Slov. 57, 565 (2007).

${ }^{17}$ M. I. Dyakonov and V. I. Perel, Phys. Lett. A 35, 459 (1971); Sov. Phys. JETP Lett. 13, 467 (1971). 
${ }^{18}$ M. Yamamoto, T. Ohtsuki, and B. Kramer, Phys. Rev. B 72, 115321 (2005).

${ }^{19}$ S. M. Badalyan and G. Vignale, Phys. Rev. Lett. 103, 196601 (2009).

${ }^{20}$ M. M. Glazov, M. A. Semina, S. M. Badalyan, and G. Vignale, Phys. Rev. B 84, 033305 (2011).

${ }^{21}$ P. Středa and P. Šeba, Phys. Rev. Lett. 90, 256601 (2003).

${ }^{22}$ S. Debald and B. Kramer, Phys. Rev. B 71, 115322 (2005).

${ }^{23}$ J. Klinovaja, P. Stano, and D. Loss, Phys. Rev. Lett. 109, 236801 (2012).

${ }^{24}$ V. L. Grigoryan, A. Matos Abiague, and S. M. Badalyan, Phys. Rev. B 80, 165320 (2009).

${ }^{25}$ S. M. Badalyan and J. Fabian, Phys. Rev. Lett. 105, 186601 (2010).

${ }^{26}$ B. Scharf, A. Matos-Abiague, and J. Fabian, Phys. Rev. B 86, 075418 (2012).
${ }^{27}$ S. Komiyama, H. Hirai, M. Ohsawa, Y. Matsuda, S. Sasa, and T. Fujii, Phys. Rev. B 45, 11085 (1992).

${ }^{28}$ S. M. Badalian, Y. B. Levinson, and D. L. Maslov, Sov. Phys. JETP Lett. 53, 619 (1991); D. L. Maslov, Y. B. Levinson, and S. M. Badalian, Phys. Rev. B 46, 7002 (1992).

${ }^{29}$ Y. Ji, Y. Chung, D. Sprinzak, M. Heiblum, D. Mahalu, and H. Shtrikman, Nature (London) 422, 415 (2003).

${ }^{30}$ T. Nakajima, K.-T. Lin, and S. Komiyama, arXiv:1207.7243.

${ }^{31}$ A. Bertoni, P. Bordone, R. Brunetti, C. Jacoboni, and S. Reggiani, Phys. Rev. Lett. 84, 5912 (2000).

${ }^{32}$ L. Chirolli, D. Venturelli, F. Taddei, R. Fazio, and V. Giovannetti, Phys. Rev. B 85, 155317 (2012).

${ }^{33}$ J. Reijniers and F. M. Peeters, J. Phys.: Condens. Matter 12, 9771 (2000).

${ }^{34}$ S. M. Badalyan and F. M. Peeters, Nanotechnology 12, 570 (2001).

${ }^{35}$ I. V. Tokatly, Phys. Rev. Lett. 101, 106601 (2008).

${ }^{36}$ Q.-F. Sun, X. C. Xie, and J. Wang, Phys. Rev. B 77, 035327 (2008). 